\title{
Room-temperature electron spin polarization exceeding $90 \%$ in an opto-spintronic semiconductor nanostructure via remote spin filtering
}

Yuqing Huang, Ville Polojarvi, Satoshi Hiura, Pontus Höjer, Arto Aho, Riku Isoaho, Teemu Hakkarainen, Mircea Guina, Shino Sato, Junichi Takayama, Akihiro Murayama, Irina Buyanova and Weimin Chen

The self-archived postprint version of this journal article is available at Linköping University Institutional Repository (DiVA):

http://urn.kb.se/resolve?urn=urn:nbn:se:liu:diva-175609

N.B.: When citing this work, cite the original publication.

Huang, Y., Polojarvi, V., Hiura, S., Höjer, P., Aho, A., Isoaho, R., Hakkarainen, T., Guina, M., Sato, S., Takayama, J., Murayama, A., Buyanova, I., Chen, W., (2021), Room-temperature electron spin polarization exceeding $90 \%$ in an opto-spintronic semiconductor nanostructure via remote spin filtering, Nature Photonics, 15, 475-482. https://doi.org/10.1038/s41566-021-00786-y

Original publication available at:

https://doi.org/10.1038/s41566-021-00786-y

Copyright: Nature Research http://www.nature.com/ 
1 Room-temperature electron spin polarization exceeding $90 \%$ in an opto-

\title{
spintronic semiconductor nanostructure via remote spin filtering
}

\author{
Yuqing Huang ${ }^{1 *}$, Ville Polojärvi², Satoshi Hiura ${ }^{3}$, Pontus Höjer ${ }^{1}$, Arto Aho ${ }^{2}$, Riku Isoaho², \\ Teemu Hakkarainen ${ }^{2}$, Mircea Guina ${ }^{2}$, Shino Sato $^{3}$, Junichi Takayama ${ }^{3}$, Akihiro Murayama ${ }^{3}$, \\ Irina A. Buyanova ${ }^{1}$ and Weimin M. Chen ${ }^{1 *}$ \\ ${ }^{1}$ Department of Physics, Chemistry and Biology, Linköping University, S-581 83 Linköping, \\ Sweden \\ ${ }^{2}$ Optoelectronics Research Centre, Physics Unit, Tampere University, Korkeakoulunkatu 3, \\ 33720 Tampere, Finland \\ ${ }^{3}$ Faculty of Information Science and Technology, Hokkaido University, Kita 14, Nishi 9, \\ Kita-ku, Sapporo 060-0814, Japan \\ *Correspondence to: weimin.chen@liu.se (W.M.C.), yuqing.huang@liu.se (Y.Q.H.)
}

Abstract An exclusive advantage of semiconductor spintronics is its potential for optospintronics that will allow integration of spin-based information processing/storage with photon-based information transfer/communications. Unfortunately, progresses have so far been severely hampered by the failure to generate nearly fully spin-polarized charge carriers in semiconductors at room temperature. Here, we demonstrate successful generation of conduction electron spin polarization exceeding $90 \%$ at room temperature without a magnetic field in a non-magnetic all-semiconductor nanostructure, which remains high even up to $110^{\circ} \mathrm{C}$. This is accomplished by remote spin filtering of InAs quantum-dot electrons via an adjacent tunneling-coupled GaNAs spin filter. We further show that the quantum-dot electron spin can be remotely manipulated by spin control in the adjacent spin filter, paving the way for remote spin encoding and writing of quantum memory as well as for remote spin control of spin-photon interfaces. This work demonstrates the feasibility to implement opto-spintronic functionality in common semiconductor nanostructures. 
Spintronics aims to explore the spin degree of freedom of the electron for information storage, processing and transfer ${ }^{1-6}$. Since $1990 \mathrm{~s}$, we have witnessed great success of metalbased spintronics that has revolutionized the mass data storage industry ${ }^{5,6}$. Recently, demonstration of ultralong spin transport and giant magneto-tunneling resistance in graphene, a well-known Van der Waals semimetal, has attracted intensive attention ${ }^{7-9}$. There has also been an enormous push for semiconductor spintronics during the past two decades ${ }^{1-4}$, with the aim to capitalize the past and current success of charge-based semiconductor technology and to make its spin counterpart the backbone of future spintronics, just like semiconductors have done in today's electronics/photonics. An exclusive advantage of semiconductor spintronics is its potential to interface with photonic devices that allows exchange of spin quantum information via photons, leading to opto-spintronic devices serving as spin-photon interfaces essential for inter-connects and data communications among and between classical and quantum information nodes and systems ${ }^{10-12}$.

As conventional semiconductors are generally non-magnetic, a prerequisite for the success of semiconductor spintronics and opto-spintronics is to generate nearly fully spinpolarized carriers in semiconductors at and above room temperature (RT) at which today's devices operate; the required maximum operating temperature is $70^{\circ} \mathrm{C}$ for commercial applications, $85^{\circ} \mathrm{C}$ for industrial applications, and $125^{\circ} \mathrm{C}$ for automotive and military applications ${ }^{13}$. As illustrated in Fig.1, numerous approaches have been attempted including spin injection into semiconductors from e.g. diluted magnetic semiconductors ${ }^{14,15}$, ferromagnetic metals ${ }^{16-20}$, Heusler alloys ${ }^{21,22}$, magnetic oxides ${ }^{23,24}$, as well as optical spin generation in semiconductors such as quantum wells, quantum dots (QDs) ${ }^{25-27}$ and most recently $2 \mathrm{D}$ transition metal dichalcogenides ${ }^{28-30}$. Despite impressive advances, two major issues remain unresolved: low spin-generation/injection efficiency and rapid thermal quenching of generated electron spin polarization - a general trend clearly evident from Fig.1. 
Up to now, these obstacles have made efficient spin generation in semiconductors at/above RT elusive, severely limiting carrier spin polarization as well as associated optical polarization to below $60 \%$ regardless of what approaches were employed (see Fig.1). Usually, steady-state electron spin polarization $\left(P_{e}=\frac{n_{\uparrow}-n_{\downarrow}}{n_{\uparrow}+n_{\downarrow}}\right)$ in a semiconductor is governed by spin-relaxation time $\tau_{s}$, lifetime $\tau$ and the initial spin polarization degree $P_{0}$ created by spin generation/injection (see Supplementary Note),

$$
P_{e}=\frac{P_{0}}{1+\tau / \tau_{s}}<P_{0}
$$

Here $n_{\uparrow}\left(n_{\downarrow}\right)$ denotes the density of the spin-up (spin-down) electrons. $P_{e}$ could be significantly lowered than $P_{0}$ by spin relaxation after spin generation, because $\tau_{s}$ is commonly much shorter than or at least comparable with $\tau$. In any case, $P_{e}$ is fundamentally limited by $P_{0}$.

To overcome this limit, an approach of defect-enabled spin filtering in a semiconductor, such as GaNAs, has recently been proposed and explored ${ }^{31-34}$. In this approach, a paramagnetic defect is introduced where its unpaired electron spin can be dynamically polarized via a spin-dependent recombination (SDR) process towards a direction defined by the helicity of circularly polarized excitation light under the optical spin orientation condition ${ }^{35}$. Such spin-polarized defect will only capture a conduction band (CB) electron with an opposite spin due to the Pauli blockade ${ }^{32}$, thereby acting as a spin filter, as illustrated in Fig.2a (also Supplementary Note). As a result, the lifetimes of the majority-spin and minority-spin are no longer equal, with the former becoming longer than the latter. $P_{e}$ now takes the modified form (see Supplementary Note)

$$
P_{e}=\frac{\left(1+P_{0}\right) \tau_{\uparrow} / \tau_{\downarrow}-\left(1-P_{0}\right)}{\left(1+P_{0}\right) \tau_{\uparrow} / \tau_{\downarrow}+\left(1-P_{0}\right)+2 \tau_{\uparrow} / \tau_{s}}
$$

Here, $\tau_{\uparrow}\left(\tau_{\downarrow}\right)$ denotes the lifetime of the spin-up (spin-down) CB electrons. Besides the dependence on $\tau_{s}, P_{e}$ also critically depends on the ratio $\tau_{\downarrow} / \tau_{\uparrow}$, as simulated in Fig.2c taking 
spin-down as the majority-spin. Even with a modest increase of $\tau_{\downarrow} / \tau_{\uparrow}$ from $1,\left|P_{e}\right|$ can easily become greater than $\left|P_{0}\right|$ - an effect equivalent to spin gain. This relaxes the otherwise strict requirement on spin relaxation as long as $\tau_{s}>\tau_{\uparrow}$, such that electrons undergoing spin flips from the majority to the minority spin will immediately be captured by the defect and annihilated with valence band (VB) holes. Thanks to thermally accelerated carrier capture and recombination via defects in semiconductors, rather high RT $P_{e}$ has been reported in GaNAs thin films, quantum wells and nanodisks ${ }^{31,32,34}$. However, the confirmed maximum $P_{e}$ remains restricted so far to below $60 \%{ }^{34}$. The main problems stem from the fact that the active region of a device, i.e. GaNAs, is also the spin-filtering region itself. High defect concentrations and strong, randomly oriented CB potential fluctuations, both are inherent to this highly mismatched alloy, lead to severe spin relaxation that strongly competes with spin filtering. The occupation of both heavy-hole (hh) and light-hole (lh) VB states at RT due to a small hhlh splitting, which give rise to opposite optical polarization when they recombine with spinpolarized CB electrons, also prevents GaNAs from applications in spin light-emitting devices and a spin-photon interface, where a pure photon state and its one-to-one correlation with electron spin are crucial.

\section{Principle of defect-enabled remote spin filtering}

To significantly boost electron spin polarization and spin-photon conversion efficiency for opto-spintronic applications, we propose here a new approach of defect-enabled remote spin filtering, in which the active region (e.g. QDs) is spatially separated from the spin filter (i.e. GaNAs) as illustrated in Fig.2b. The remote spin filtering is expected to be vastly superior over the standard spin filtering in a number of key aspects. First, it will reduce the risk of potential side effects induced by defects that could harm device applications by e.g. accelerating CB spin relaxation and lowering quantum efficiency or carrier mobility. More 
importantly, it will allow 2-step spin filtering when the electron ground state (e0) of the QDs is carefully tuned between the occupation levels of the majority and minority spins in GaNAs as illustrated in Fig.2b in a simplified picture. In the $1^{\text {st }}$-step, $\mathrm{CB}$ electron spins are filtered in GaNAs just like the standard single-step spin filtering. In the $2^{\text {nd }}$-step, the majority spins in GaNAs will be more favored to inject to QDs than the minority spins due to a higher quasiFermi level. Meanwhile, the minority spins are favored for back transfer from QDs to GaNAs where they will be further filtered out, leading to $\tau_{\uparrow}^{Q D}<\tau_{\downarrow}^{Q D}$ that drives the $2^{\text {nd }}$-step spin filtering. These favorable conditions provide the foundation for an even higher $P_{e}^{Q D}$ after the $2^{\text {nd }}$-step spin filtering (see Fig.2c,d).

\section{Nanostructure design for remote spin filtering}

To experimentally examine the feasibility of the proposed remote spin filtering, we have employed a semiconductor nanostructure (referred to as QD/GaNAs) consisting of InAs QDs that are tunneling coupled to a GaNAs spin-filtering layer through a thin GaAs barrier (Supplementary Fig.S1). N compositions of GaNAs over the range of 1-2.6\% were selected, which is theoretically predicted to align the GaNAs CB edge in the vicinity of the QD e0 level for resonant electron tunneling between them (see Supplementary Note). For a direct comparison, a reference QD structure (ref-QD) and reference GaNAs layers (denoted as refGaNAs) are also studied. A detailed description of the studied nanostructures and their structural characterization is given in Methods and Supplementary Figs.S1-S3.

Here we have chosen QDs as the active region of such an opto-spintronic nanostructure because they have long been recognized as being among the most promising candidate structures for spintronics due to their suppressed carrier spin relaxation/decoherence ${ }^{36}$. QDs are also known for their superior optical properties highly desirable for single photon sources ${ }^{37}$, efficient light-emitting devices ${ }^{38}$, etc. Moreover, a pure photon state and the one- 
to-one correlation between $P_{e}^{Q D}$ and circularly polarized optical polarization $P_{o p t}^{Q D}$, i.e. $P_{e}^{Q D}=$ $-P_{\text {opt }}^{Q D}$, can be fulfilled in strained InAs/GaAs QDs thanks to the fact that only the hh0 state is occupied due to a large hh-lh splitting. These merits make such QDs excellent candidates for spin-photon interfaces bridging spintronics and photonics.

\section{Spin generation at and above $\mathrm{RT}$ via remote spin filtering}

To evaluate $P_{e}^{Q D}$ resulting from the remote spin filtering, we have conducted polarization-resolved photoluminescence (PL) studies of the coupled QD/GaNAs structures under optical excitation with continuous-wave (cw) $\sigma^{+}$polarized excitation above the GaNAs bandgap (see Fig.3a). The relevant optical excitation and emission processes are illustrated in Fig.3b. Governed by the optical selection rule of the band-to-band (BB) transitions in GaNAs shown in Fig.3c, the $\sigma^{+}$excitation is expected to generate a spin imbalance among CB electrons favoring the spin-down orientation. Due to severe spin losses during the optical spin generation and the subsequent energy relaxation, however, the initial $\mathrm{CB}$ electron spin polarization $P_{0}^{\text {GaNAs }}$ is $<10 \%$ (see Supplementary Note), ${ }^{39}$ substantially lower than the theoretical limit of $50 \%$ allowed by the selection rule. Nevertheless, it provides the impetus for dynamic spin polarization of the defects and its resulting spin filtering of $\mathrm{CB}$ electrons. In Fig.3d, we show PL spectra obtained at RT from the QD/GaNAs structure with $[N]=1.5 \%$, where $\sigma^{+} / \sigma^{+}$stands for $\sigma^{+}$-excitation $/ \sigma^{+}$-detection and $\sigma^{+} / \sigma^{-}$for $\sigma^{+}$-excitation $/ \sigma^{-}$detection. They are composed of a low-energy PL band from the InAs QDs and a high-energy band from GaNAs. The latter consists of the BB transitions involving the hh and lh states with opposite PL polarization $\left(P_{P L}\right)$, see Fig.3b,c, split under the in-plane tensile strain leaving the lh emission at the lower energy. The PL and $P_{P L}$ spectra from the GaNAs BB emission of the QD/GaNAs structure are nearly identical to that from the ref-GaNAs sample (Fig.3e), indicating similar spin filtering in GaNAs. The QDs in QD/GaNAs, on the other hand, exhibit 
remarkably higher $P_{P L}^{Q D}$ of $90 \%$ at RT (i.e. $-90 \% P_{e}^{Q D}$ ) than only $2 \% P_{P L}$ (thus $-2 \% P_{e}$ ) from ref-QD shown in Fig.3f, i.e. by a factor of 45, demonstrating an extremely high efficiency of the remote spin filtering. Such high $\left|P_{e}^{Q D}\right|$ is shown to be robust against rising temperature up to $110^{\circ} \mathrm{C}(383 \mathrm{~K})$ as seen in Supplementary Fig.S5. The observed increase of $\left|P_{e}^{Q D}\right|$ from 100 $\mathrm{K}$ to RT, see Fig.1, demonstrates that the remote spin filtering is thermally activated owing to thermally accelerated capture of carriers by the spin-filtering defects - a common property for deep-level defects in semiconductors.

\section{Evidence for remote spin filtering}

As described above, a fingerprint of the spin filtering is a longer lifetime of the majority spin than the minority spin, i.e. $\tau_{\downarrow}^{Q D}>\tau_{\uparrow}^{Q D}$ under $\sigma^{+}$excitation. To verify that the observed high $\left|P_{e}^{Q D}\right|$ is indeed induced by the remote spin filtering, we performed timeresolved PL (tr-PL) studies of optical spin generation dynamics under pulsed $\sigma^{+}$excitation. In the absence of the remote spin filtering when $\tau_{\uparrow}^{Q D}=\tau_{\downarrow}^{Q D}=\tau^{Q D}$, the total decay time of the majority spin should always be shorter than the minority spin as the former additionally suffers spin flips to the latter. This is consistent with the initial shorter decay time of the $\sigma^{+}$ polarized PL than the $\sigma^{-}$-polarized PL, i.e. $\frac{\tau^{Q D}\left(\sigma^{+} / \sigma^{+}\right)}{\tau^{Q D}\left(\sigma^{+} / \sigma^{-}\right)}<1$, and the resulting $P_{P L}^{Q D}$ decrease observed in ref-QD over the time window of spin relaxation, marked by the grey area in Fig.4a. The deduced $\tau_{s}^{Q D}=80 \mathrm{ps}$ is typical for strained InAs QDs ${ }^{40}$. After spin relaxation is completed, both PL components approach towards an identical decay time $\tau^{Q D}=0.9 \mathrm{~ns}$, typical for radiative recombination in III-V QDs ${ }^{40}$. The large ratio of $\tau^{Q D} / \tau_{s}^{Q D}=11.25$ explains the weak $\left|P_{e}^{Q D}\right|$ of only $2 \%$ obtained in ref-QD under the cw excitation (Fig.3f), as $P_{e}^{Q D} \approx 0.08 P_{0}^{Q D}$ following equation (1). In sharp contrast, the initial decay of the majority- 
spin PL is slower than the minority-spin PL in QD/GaNAs, yielding $\frac{\tau^{Q D}\left(\sigma^{+} / \sigma^{+}\right)}{\tau^{Q D}\left(\sigma^{+} / \sigma^{-}\right)}>1$ over the time period when $P_{P L}^{Q D}$ drastically rises (see the grey area in Fig.4a). This is possible only if $178 \tau_{\downarrow}^{Q D}$ is much longer than $\tau_{\uparrow}^{Q D}$ to a degree that can even overcompensate spin relaxation. This 179 provides direct and unambiguous evidence for the remote spin filtering as being the physical mechanism for the remarkably high $\left|P_{e}^{Q D}\right|$ of $90 \%$ observed at RT in the QD/GaNAs structure.

Another hallmark of the defect-enabled spin filtering is that the PL intensity with spin filtering should be higher than that without spin filtering thanks to spin blockade of defectmediated recombination losses of the majority-spin electrons in the former. In other words, the ratio between the total PL intensities under circularly $\left(\sigma^{+}\right.$or $\left.\sigma^{-}\right)$and linearly $\left(\sigma^{x}\right)$ polarized optical excitation - the so-called SDR ratio, $\frac{I_{P L}\left(\sigma^{ \pm} \text {exc. }\right)}{I_{P L}\left(\sigma^{x} \text { exc. }\right)}$, should be 1 without spin filtering but $>1$ with spin filtering. This effect is predicted from the rate equation analysis described in Supplementary Note and Fig.S10. Each step of spin filtering is expected to exhibit a step-wise increase of the SDR ratio above 1. Indeed, the SDR ratio was experimentally shown to be about 1.63 by monitoring the GaNAs PL and 1.87 by monitoring as an additional, direct proof of the 2-step remote spin filtering. Further support for the defect-enabled remote spin filtering is provided by the quenching of the SDR ratio in a transverse magnetic field by monitoring the QD PL, see Supplementary Fig.S15, due to spin precession and the resulting spin depolarization of the defect electrons in GaNAs that deactivates spin filtering. An effective spin lifetime $T_{S}=592 \mathrm{ps}$ is deduced from a linewidth analysis with $T_{s}=\hbar /\left(\mu_{B} g B_{1 / 2}\right)$, where $B_{1 / 2}=9.6 m T$ is the half-width of the Lorentzian line fitting ${ }^{35}$. This value is consistent with the spin lifetime of the spin-filtering $G a_{i}^{2+}$ defects in GaNAs ${ }^{33}$. 


\section{Effect of barrier thickness and energy-level alignment}

To identify an optimal design of the coupled QD/GaNAs nanostructures for spin generation, we have investigated a series of QD/GaNAs structures with different GaAs barrier thicknessed $d$. As shown in the lower panel of Fig.4b, the QD $\left|P_{e}^{Q D}\right|$ increases with decreasing $d$, which is correlated with the shortening of the corresponding QD PL decay time, denoted by $\tau^{Q D}\left(\sigma^{x} / \sigma^{x}\right)$, obtained under linearly polarized excitation and detection (see Supplementary Fig.S9). The observation of a nearly exponential increase of $\tau^{Q D}\left(\sigma^{x} / \sigma^{x}\right)$ with increasing $d$ indicates quantum tunneling as the nature of electron transfer between QDs and GaNAs. The increase in $\left|P_{e}^{Q D}\right|$ with decreasing $d$ is also found to correlate with an increase in the ratio $210 \frac{\tau^{Q D}\left(\sigma^{+} / \sigma^{+}\right)}{\tau^{Q D}\left(\sigma^{+} / \sigma^{-}\right)}$(correlated with $\left.\tau_{\downarrow}^{Q D} / \tau_{\uparrow}^{Q D}\right)$, see the upper panel of Fig. $4 \mathrm{~b}$, which further proves the 211 dominant role of the remote spin filtering in the achieved high $\left|P_{e}^{Q D}\right|$. In the case of $212 \frac{\tau^{Q D}\left(\sigma^{+} / \sigma^{+}\right)}{\tau^{Q D}\left(\sigma^{+} / \sigma^{-}\right)}>1$, the spin filtering dominates over spin relaxation, leading to spin gain as marked by the red area in Fig.4b. The optimal value of $d$ for spin filtering is found to be within the range of 3-5 $\mathrm{nm}$. The fact that the observed $\left|P_{e}^{Q D}\right|=90 \%$ in QD/GaNAs with $d=3$ $\mathrm{nm}$ is higher than the maximum value $P_{e}^{Q D} \approx 75 \%$ allowed in the absence of spin filtering, according to equation (1) using $\tau^{Q D}=26 \mathrm{ps}$ (Supplementary Fig.S9) and $\tau_{s}^{Q D}=80 \mathrm{ps}$ (Fig.4a), 217 is a further proof of the remote spin filtering.

To examine the effect of energy-level alignment on spin generation, we investigated a set of $\mathrm{QD} / \mathrm{GaNAs}$ coupled structures with varying $\mathrm{N}$ concentrations between $1 \%$ and $2.6 \%$, which shifts the GaNAs $\mathrm{CB}$ edge ${ }^{41}$ and thus its alignment with the QD e0 level. The maximum $\left|P_{e}^{Q D}\right|=90 \%$ is found for $[\mathrm{N}]=1.5 \%$, where the maximum overlap is reached between the density of state of the GaNAs and QD as described in Supplementary Note. 


\section{Remote spin control enabled by remote spin filtering}

Below we shall show that $\mathrm{QD} P_{e}^{Q D}$ in $\mathrm{QD} / \mathrm{GaNAs}$ can be remotely manipulated at $\mathrm{RT}$ by controlling electron spins in GaNAs. For example, by driving spin precession of GaNAs $\mathrm{CB}$ electrons in an applied transverse magnetic field $B$, as depicted in Fig.5a, their spin orientation will alternate between spin-up and spin-down directions at a Larmor frequency $v=|g| \mu_{B} B / h$. Indeed, as shown in Fig.5b, the measured transient $P_{P L}^{G a N A s}$ oscillates at $v=$ $2.3 \mathrm{GHz}$ at $B=164 \mathrm{mT}$ and $5.2 \mathrm{GHz}$ at $B=380 \mathrm{mT}$. The fitting of the $v \sim B$ relation yields $|g| \approx 1$, see Fig.5c, which agrees with the CB electron g-factor of $\mathrm{GaN}_{0.015} \mathrm{As}_{0.985}{ }^{31,42}$.

Strikingly, the same oscillations are also observed in $P_{P L}^{Q D}$ with no apparent phase delay, but at a larger amplitude. The observed cloning demonstrates the capability of very fast remote spin manipulation of QD electrons by controlling CB electrons in GaNAs. The effect of electron spin precession of the spin-filtering $G a_{i}^{2+}$ defects $(g=2){ }^{32}$ on QD $P_{e}^{Q D}$ can be revealed in Hanle measurements ${ }^{7,20,43}$ under cw optical excitation (see Supplementary Note). Both Hanle curves obtained by detecting $\left|P_{P L}^{Q D}\right|$ and $\left|P_{P L}^{G a N A s}\right|$ at RT, shown in Fig.5d-e, can be fitted by a Lorentzian line-shape ${ }^{35}$ with an identical electron spin lifetime $T_{s}=\hbar /\left(\mu_{B} g B_{1 / 2}\right)=196 \mathrm{ps}$, consistent with the reported electron spin lifetime of the spin-filtering $G a_{i}^{2+}$ defects under an intermediate excitation density ${ }^{33}$. This demonstrates that $P_{e}^{Q D}$ and $P_{P L}^{Q D}$ can be remotely manipulated by spin control of both defect and CB electrons in GaNAs, relevant to remote spin encoding and writing of quantum memory in QDs. The above results in turn also show the potential of using the QD light emitters to remotely readout the information encoded in GaNAs CB and defect electron spins and to convert the spin-coded information to polarized photons for information transfer and communications.

It is worth noting that the following two striking and extraordinary features have made the remote spin filtering approach attractive and standout. The first feature is the built-in spin gain/amplification function, which effectively removes the seemingly "impossible" 
simultaneous requirements of (a) perfect spin generation/injection and (b) virtually without

250

251

252

253

254

255

256

257

258

259

260

261

262

263

264

265 any subsequent spin loss imposed on other traditional methods in order to achieve near $100 \%$ $P_{e}$. For example, $90 \% P_{e}$ can be achieved in our work despite of the fact that the initial CB electron spin polarization $P_{0}$ generated by optical orientation in GaNAs is $<10 \%$. In contrast, spin polarization in several spin aligners as well as optical orientation in TMDC is capable of generating $P_{0}$ of near $100 \%$; yet, $P_{e}$ reported to date never exceeds $60 \%$ at $\mathrm{RT}^{28}$ The second feature of our approach is the overall trend of increasing $P_{e}$ with increasing temperature as illustrated in Fig.1, thanks to the thermally activated and accelerated spin-filtering process, which is opposite to the detrimental trend seen in all other known methods. The combination of these two features has, for the first time, enabled us to achieve steadily high $P_{e}$ (around $90 \%$ ) at/above RT, paving the way for practical applications of future semiconductor spintronics and opto-spintronics.

The principle of our proposed approach is expected to be valid also for electrical spin injection as long as injected electrons possess non-vanishing spin polarization that can trigger the defect-enabled spin filtering. We should also point out that optical spin generation is of direct relevance to device applications in future opto-spintronics and spin-photonics, such as optically pumped spin-LEDs and spin lasers, optical initiation, manipulation and readout of spin qubits, and quantum communications and computation based on spin-photon interfaces in all-optical devices and systems in general.

In summary, we have proposed a novel approach of defect-enabled, two-step remote spin filtering and have demonstrated its capability to generate electron spin polarization of $90 \%$ at RT in an opto-spintronic semiconductor nanostructure, which remains steadily high even up to $110^{\circ} \mathrm{C}$. This represents the highest $\mathrm{RT}$ electron spin polarization ever reported in a semiconductor, which is a giant leap from the previously reported highest value of $60 \%$ in any semiconductor by any approach. We further show that QD electron spin can be manipulated 
by spin control of both conduction and defect electrons in the adjacent spin filter, providing new opportunities for remote spin encoding and readout at RT that will utilize both conduction and defect electron spins. The fact that the demonstrated opto-spintronic functionality is implemented in a commonly used semiconductor nanostructure system (i.e. InAs/GaAs QDs) based on the mature GaAs technology can greatly facilitate the integration of spin functionalities with the existing electronic and photonic devices. It could also pave the way for a range of potential spintronic and opto-spintronic applications exploiting the stateof-the-art GaAs technology platform, such as spin-LEDs, spin lasers, spin-polarized singlephoton sources, spin-photon interfaces, spin qubits, etc.

\section{References:}

1. Wolf, S. A. Spintronics: A Spin-Based Electronics Vision for the Future. Science 294, $1488-1495$ (2001).

2. Žutić, I., Fabian, J. \& Das Sarma, S. Spintronics: Fundamentals and applications. Rev. Mod. Phys. 76, 323-410 (2004).

3. Awschalom, D. D. \& Flatté, M. E. Challenges for semiconductor spintronics. Nature Phys. 3, 153-159 (2007).

4. Dietl, T., Awschalom, D. D., Kaminska, M., Ohno, H. Spintronics (Academic Press, Cambridge, Massachusetts, US, 2008).

5. Chappert, C., Fert, A. \& Nguyen Van Dau, F. The emergence of spin electronics in data storage. Nature Mater. 6, 813-823 (2007).

6. Parkin, S.S., Hayashi M., Thomas L. Magnetic Domain-Wall Racetrack Memory. Science 320, 190-194 (2008).

7. Tombros, N., Jozsa, C., Popinciuc, M., Jonkman, H. T. \& van Wees, B. J. Electronic spin transport and spin precession in single graphene layers at room temperature. Nature $\mathbf{4 4 8 ,}$ 
571-574 (2007).

300

301

302

303

304

305

306

307

8. Gurram, M., Omar, S. \& Wees, B. J. van. Bias induced up to $100 \%$ spin-injection and detection polarizations in ferromagnet/bilayer-hBN/graphene/hBN heterostructures. $N a t$. Commun. 8, 248 (2017).

9. Song, T. et al. Giant tunneling magnetoresistance in spin-filter van der Waals heterostructures. Science 360, 1214-1218 (2018).

10. Javadi, A. et al. Spin-photon interface and spin-controlled photon switching in a nanobeam waveguide. Nat. Nanotechnol. 13, 398-403 (2018).

11. Luo, Z. et al. A Spin-Photon Interface Using Charge-Tunable Quantum Dots Strongly Coupled to a Cavity. Nano Lett. 19, 7072-7077 (2019).

12. Berezovsky, J. et al. Nondestructive Optical Measurements of a Single Electron Spin in a Quantum Dot. Science 314, 1916-1920 (2006).

13. Wikipedia contributors, Operating temperature. Wikipedia, https://en.wikipedia.org/w/index.php?title=Operating_temperature\&oldid=957567425 (2020).

14. Jonker, B. T. et al. Quantifying electrical spin injection: Component-resolved electroluminescence from spin-polarized light-emitting diodes. Appl. Phys. Lett. 79, 3098-3100 (2001).

15. Fiederling, R. et al. Injection and detection of a spin-polarized current in a light-emitting diode. Nature 402, 787-790 (1999).

16. Hanbicki, A. T., Jonker, B. T., Itskos, G., Kioseoglou, G. \& Petrou, A. Efficient electrical spin injection from a magnetic metal/tunnel barrier contact into a semiconductor. Appl. Phys. Lett. 80, 1240-1242 (2002).

17. Zhu, H. J. et al. Room-Temperature Spin Injection from Fe into GaAs. Phys. Rev. Lett. 87, 016601 (2001). 
18. Jiang, X. et al. Highly Spin-Polarized Room-Temperature Tunnel Injector for Semiconductor Spintronics using MgO(100). Phys. Rev. Lett. 94, 056601 (2005).

19. Cadiz, F. et al. Electrical Initialization of Electron and Nuclear Spins in a Single Quantum Dot at Zero Magnetic Field. Nano Lett. 18, 2381-2386 (2018).

20. Dash, S. P., Sharma, S., Patel, R. S., de Jong, M. P. \& Jansen, R. Electrical creation of spin polarization in silicon at room temperature. Nature 462, 491-494 (2009).

21. Dong, X. Y. et al. Spin injection from the Heusler alloy $\mathrm{Co}_{2} \mathrm{MnGe}$ into Al ${ }_{0.1} \mathrm{Ga}_{0.9} \mathrm{As} / \mathrm{GaAs}$ heterostructures. Appl. Phys. Lett. 86, 102107 (2005).

22. Ramsteiner, M. et al. $\mathrm{Co}_{2} \mathrm{FeSi} / \mathrm{GaAs} /(\mathrm{Al}, \mathrm{Ga}) \mathrm{As}$ spin light-emitting diodes: Competition between spin injection and ultrafast spin alignment. Phys. Rev. B 78, 121303 (2008).

23. Chen, J.-Y., Wong, T.-M., Chang, C.-W., Dong, C.-Y. \& Chen, Y.-F. Self-polarized spinnanolasers. Nat. Nanotechnol. 9, 845-850 (2014).

24. Chen, J. Y. et al. Efficient Spin-Light Emitting Diodes Based on InGaN/GaN Quantum Disks at Room Temperature: A New Self-Polarized Paradigm. Nano Lett. 14, 3130-3137 (2014).

25. Kroutvar, M. et al. Optically programmable electron spin memory using semiconductor quantum dots. Nature 432, 81-84 (2004).

26. Urbaszek, B. et al. Efficient dynamical nuclear polarization in quantum dots: Temperature dependence. Phys. Rev. B 76, 201301 (2007).

27. Beyer, J., Buyanova, I. A., Suraprapapich, S., Tu, C. W. \& Chen, W. M. Strong roomtemperature optical and spin polarization in InAs/GaAs quantum dot structures. Appl. Phys. Lett. 98, 203110 (2011).

28. Zhu, B., Zeng, H., Dai, J., Gong, Z. \& Cui, X. Anomalously robust valley polarization and valley coherence in bilayer WS2. Proc. Natl. Acad. Sci. 111, 11606-11611 (2014). 29. Dhall, R. et al. Strong Circularly Polarized Photoluminescence from Multilayer $\mathrm{MoS}_{2}$ 
Through Plasma Driven Direct-Gap Transition. ACS Photonics 3, 310-314 (2016).

30. Zeng, H., Dai, J., Yao, W., Xiao, D. \& Cui, X. Valley polarization in MoS2 monolayers by optical pumping. Nat. Nanotechnol. 7, 490-493 (2012).

31. Kalevich, V. K. et al. Spin-dependent electron dynamics and recombination in $\mathrm{GaAs}_{1-\mathrm{x}} \mathrm{N}_{\mathrm{x}}$ alloys at room temperature. JETP Lett. 85, 174-178 (2007).

32. Wang, X. J. et al. Room-temperature defect-engineered spin filter based on a nonmagnetic semiconductor. Nat. Mater. 8, 198-202 (2009).

33. Puttisong, Y., Buyanova, I. A. \& Chen, W. M. Limiting factor of defect-engineered spinfiltering effect at room temperature. Phys. Rev. B 89, 195412 (2014).

34. Chen, S. et al. Room-temperature polarized spin-photon interface based on a semiconductor nanodisk-in-nanopillar structure driven by few defects. Nat. Commun. 9, 3575 (2018).

35. Meier, F., Zakharchenya, B. P. Optical Orientation (North Holland, Amsterdam, 1984).

36. Awschalom, D. D., Bassett, L. C., Dzurak, A. S., Hu, E. L. \& Petta, J. R. Quantum Spintronics: Engineering and Manipulating Atom-Like Spins in Semiconductors. Science 339, 1174-1179 (2013).

37. Senellart, P., Solomon, G. \& White, A. High-performance semiconductor quantum-dot single-photon sources. Nat. Nanotechnol. 12, 1026-1039 (2017).

38. Yang, Y. et al. High-efficiency light-emitting devices based on quantum dots with tailored nanostructures. Nat. Photonics 9, 259-266 (2015).

39. Puttisong, Y. et al. Room-Temperature Electron Spin Amplifier Based on Ga(In)NAs Alloys. Adv. Mater. 25, 738-742 (2013).

40. Marcinkevičius, S., Siegert, J. \& Zhao, Q. X. Carrier spin dynamics in modulation-doped InAs/GaAs quantum dots. J. Appl. Phys. 100, 054310 (2006).

41. Bi, W. G. \& Tu, C. W. Bowing parameter of the band-gap energy of GaNxAs1-x. Appl. 
Phys. Lett. 70, 1608-1610 (1997).

42. Pryor, C. E. \& Flatté, M. E. Landé g Factors and Orbital Momentum Quenching in Semiconductor Quantum Dots. Phys. Rev. Lett. 96, 026804 (2006).

43. Hanbicki, A. T., Jonker, B. T., Itskos, G., Kioseoglou, G. \& Petrou, A. Efficient electrical spin injection from a magnetic metal/tunnel barrier contact into a semiconductor. Appl. Phys. Lett. 80, 1240-1242 (2002).

44. Aho, A., Korpijärvi, V., Tukiainen, A., Puustinen, J., Guina, M. Incorporation model of $\mathrm{N}$ into GaInNAs alloys grown by radio-frequency plasma-assisted molecular beam epitaxy. J. Appl. Phys. 116, 213101 (2014).

45. Gladysiewicz, M., Kudrawiec, R., Wartak, M.S. Theoretical studies of optical gain tuning by hydrostatic pressure in GaInNAs/GaAs quantum wells. J. Appl. Phys. 115, 033515 (2014).

\section{Acknowledgments}

W.M.C. acknowledges support from the Swedish Research Council (Grant No. 2016-05091, 2020-04530) and from the Swedish Foundation for International Cooperation in Research and Higher Education (STINT) (Grant No. JA2014-5698); I.A.B. from the Swedish Research Council (Grant No. 2015-05532, 2019-04312); W.M.C. and I.A.B. from Linköping University through the Professor Contracts and the Swedish Government Strategic Research Area in Materials Science on Functional Materials at Linköping University (Faculty Grant SFO-MatLiU \# 2009-00971); M.G. from the European Research Council, ERC AdG AMETIST (Grant No. \#695116) and from the Academy of Finland, NanoLight project (Grant No. \#310985); T.H. from the Academy of Finland QuantSi project (Grant No. \#323989); A.M. from Japan Society for the Promotion of Science (JSPS) (Grant Numbers 16H06359, 19H05507, and bilateral program); S.H. from JSPS (Grant Number 19K15380). M.G. acknowledges 
399 Marianna Raappana for AFM characterization, and Elina Anttola for samples preparation.

400

401 Author contributions

402 W.M.C. conceived and coordinated the project. Y.Q.H. and P.J. conducted cw optical and 403 magnetooptical experiments and analyzed the data under the supervision of W.M.C. and 404 I.A.B. The fabrication process of the experimental samples was developed by V.P., A.A., and 405 T.H. under the supervision of M.G. Epitaxy and XRD characterization was performed by R.I. 406 and A.A. S.H., S.S., J.T. and Y.Q.H. performed time-resolved optical and magnetooptical 407 experiments and analyzed data under the supervision of A.M., I.A.B. and W.M.C. Y.Q.H. and 408 W.M.C. wrote the manuscript, with contributions from all other coauthors.

409

410 Competing interests

411 The authors declare no competing interests. 


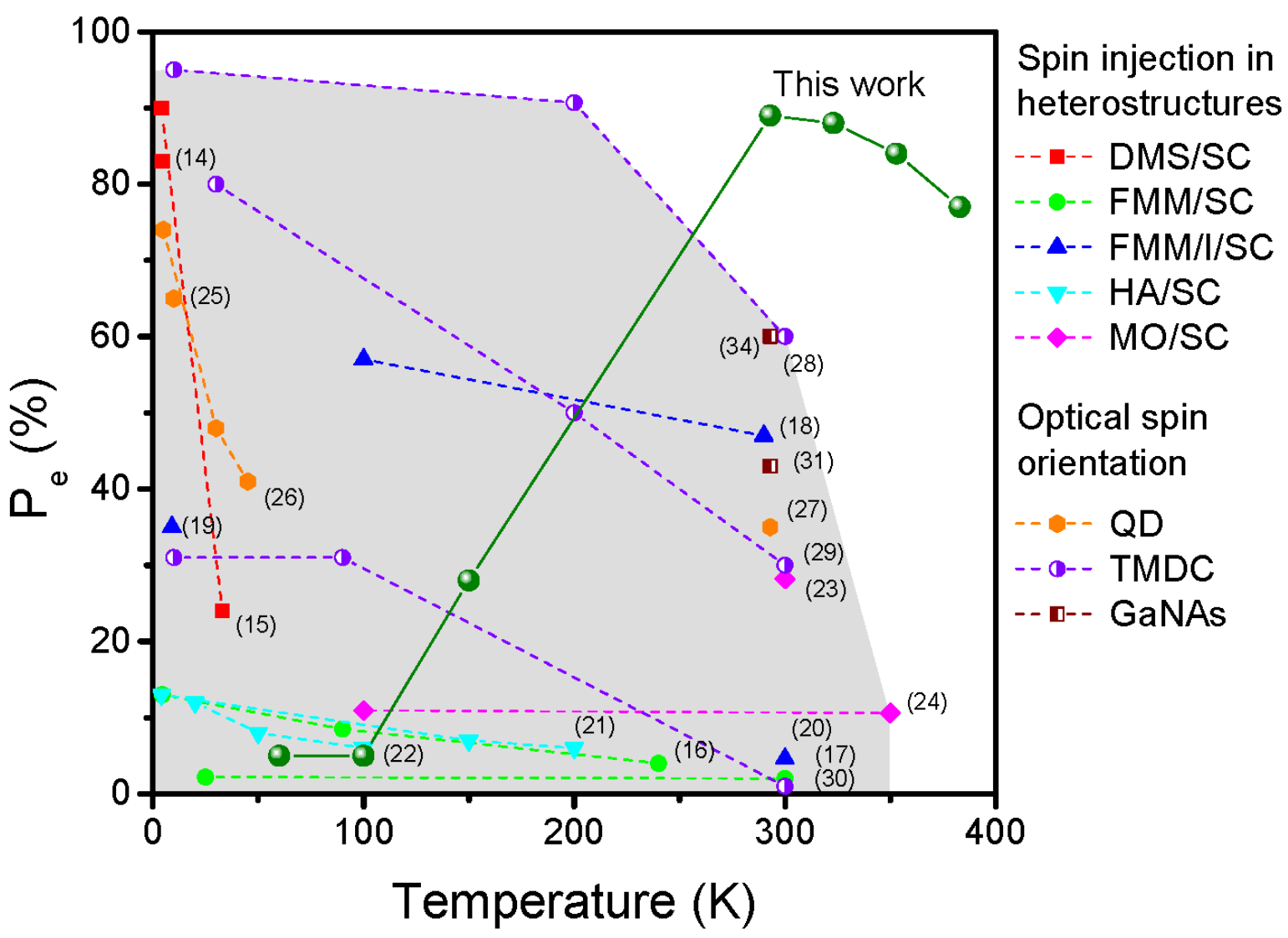

414 Fig. 1 | State-of-art spin generation in semiconductor materials. The steady-state conduction electron spin polarization $P_{e}$ achieved in this work in comparison with representative $P_{e}$ values reported in the literature in semiconductors (SC) through spin injection from dilute magnetic semiconductors (DMS), ferromagnetic metals (FMM),

418 ferromagnetic metal/insulator (FMM/I), Heusler alloys (HA), magnetic oxides (MO) and from 419 optical orientation in QDs and 2D transition metal dichalcogenides (TMDC) and spin filtering in GaNAs. Each dashed line shows the temperature dependence of $P_{e}$ for a given material system. The grey shaded area indicates the range of the measured $P_{e}$ and measurement temperature where earlier studies have been restricted to. 
a
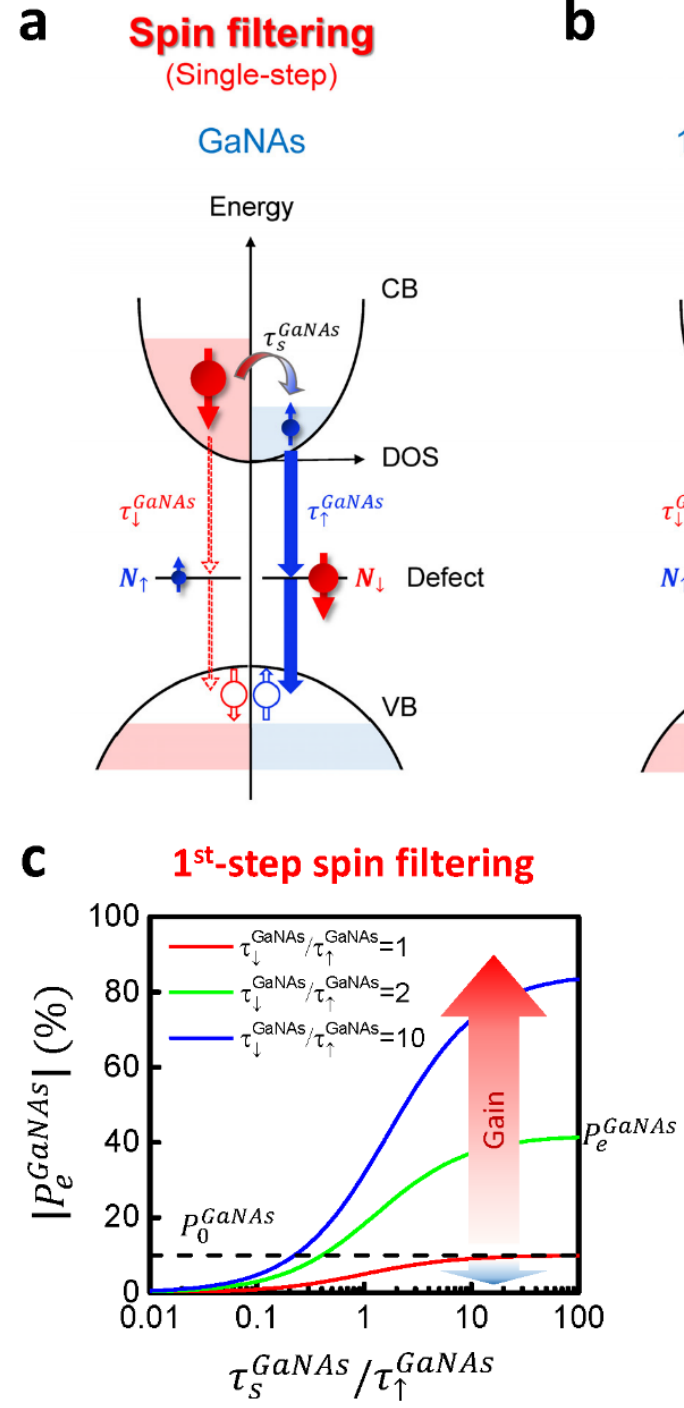

b Remote Spin filtering

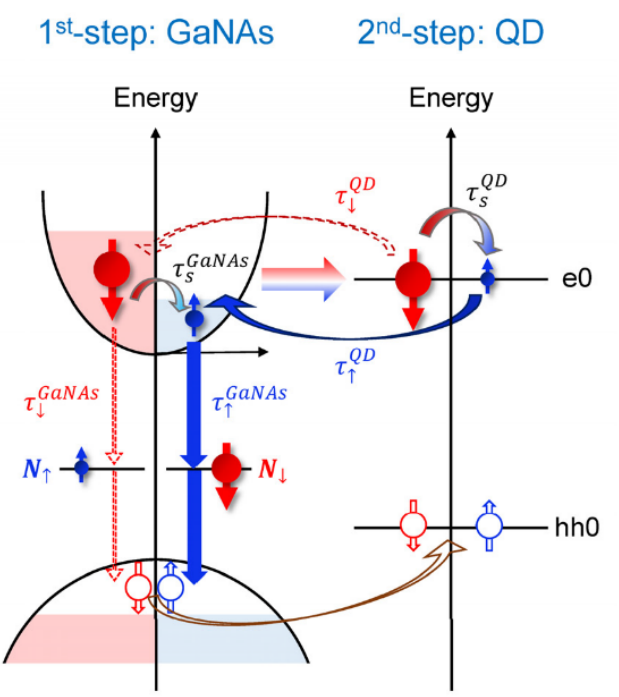

d $\quad 2^{\text {nd }}$-step spin filtering

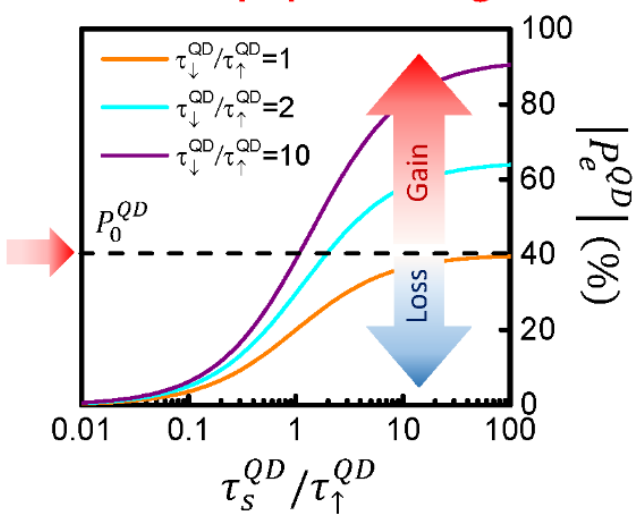

Fig. 2 Principle of the defect-enabled remote spin filtering. a, A simplified picture of the working principle of the single-step defect-enabled spin filtering in GaNAs, and $\mathbf{b}$, the 2-step remote spin filtering in a coupled QD/GaNAs nanostructure. DOS denotes the density of state. $N_{\uparrow}\left(N_{\downarrow}\right)$ refers to the spin-up (spin-down) electron density of the spin-filtering defect. c,d, Simulations of electron spin polarization enhancement as a result of the $1^{\text {st }}$-step and $2^{\text {nd }}$ step spin filtering, demonstrating the capability of sizable spin gain during each step once the ratio of the lifetimes between the majority spin and minority spin $\left(\tau_{\downarrow} / \tau_{\uparrow}\right)$ becomes greater than 1 . The horizontal dashed lines mark the initial spin polarization in each step. The illustration and the simulations are done by taking, as an example, spin-down as the majority spin. The same conclusion can be drawn when the majority spin is spin-up, by interexchanging the two spin orientations and reversing the sign of the spin polarization. 
a

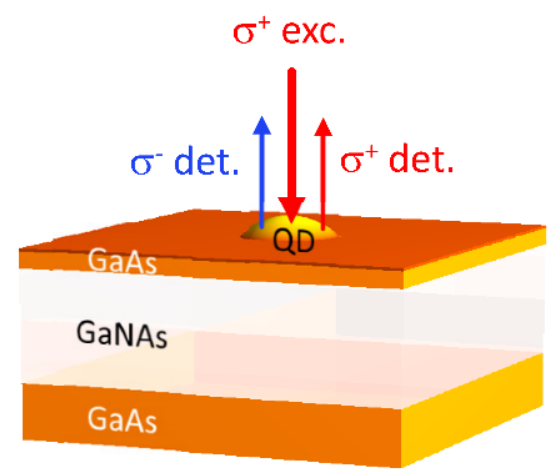

b

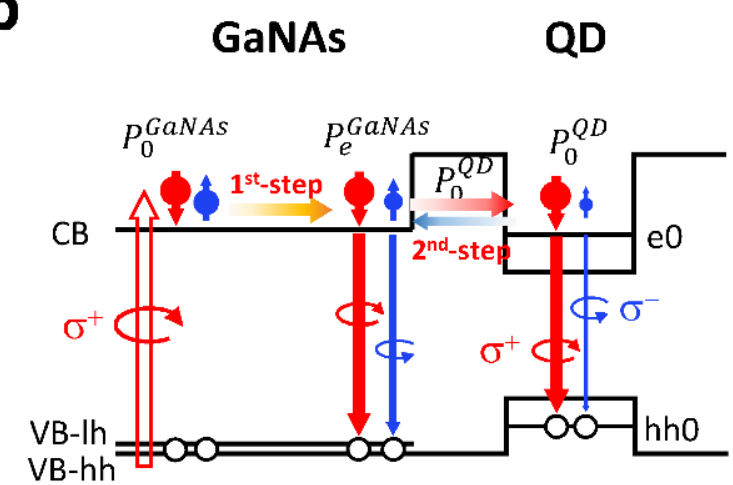

C

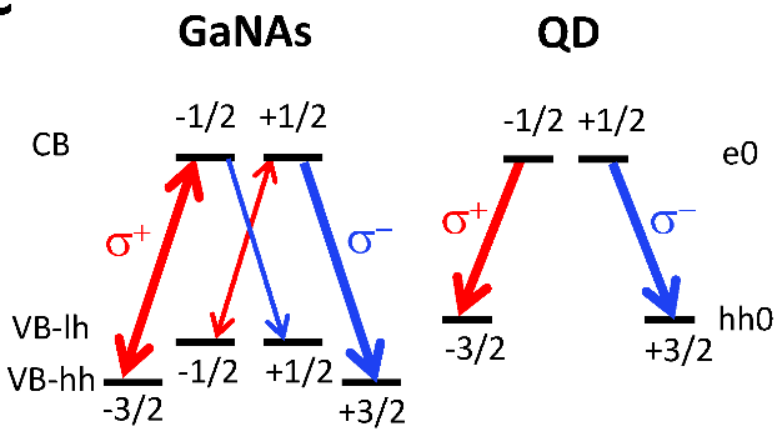

d Photon Energy (eV)
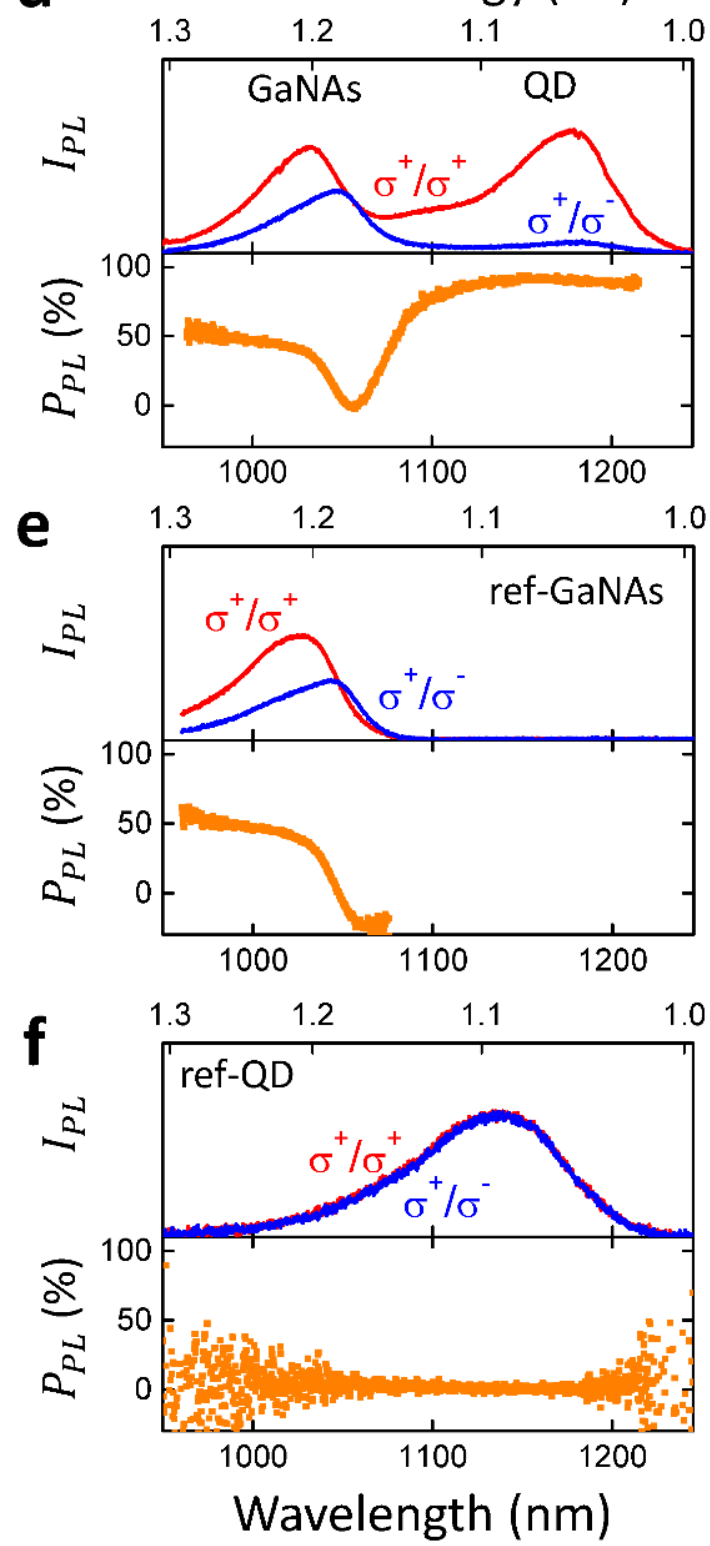

\section{Fig. 3 Generation of record-high electron spin polarization at RT via remote spin}

filtering. a, A schematic illustration of the experimental configuration for polarizationresolved photoluminescence, employed to optically generate and detect electron spin polarization. b, Energy diagram of the coupled QD/GaNAs structure and the relevant optical transitions. c, Optical selection rules of the BB transitions in GaNAs and QDs. d-f, PL and $P_{P L}$ spectra measured at RT under $\sigma^{+}$excitation from the QD/GaNAs structure (d), the refGaNAs sample (e), and the ref-QD sample (f). The excitation wavelength was $920 \mathrm{~nm}$ for the QD/GaNAs structure and the ref-GaNAs sample, and $850 \mathrm{~nm}$ for the ref-QD sample. 
a

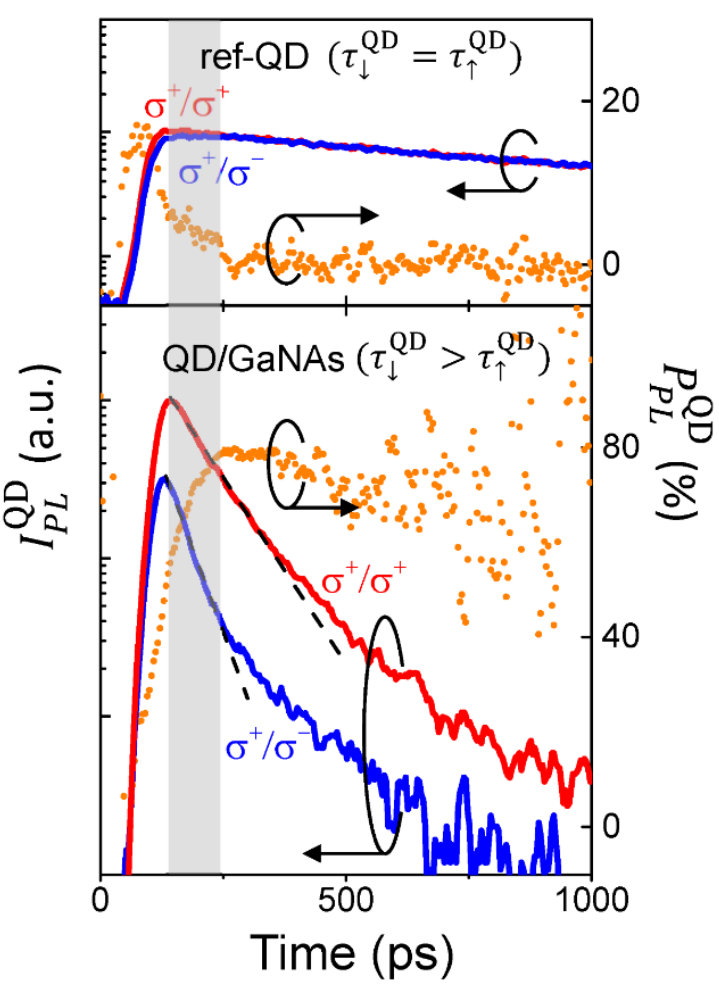

b

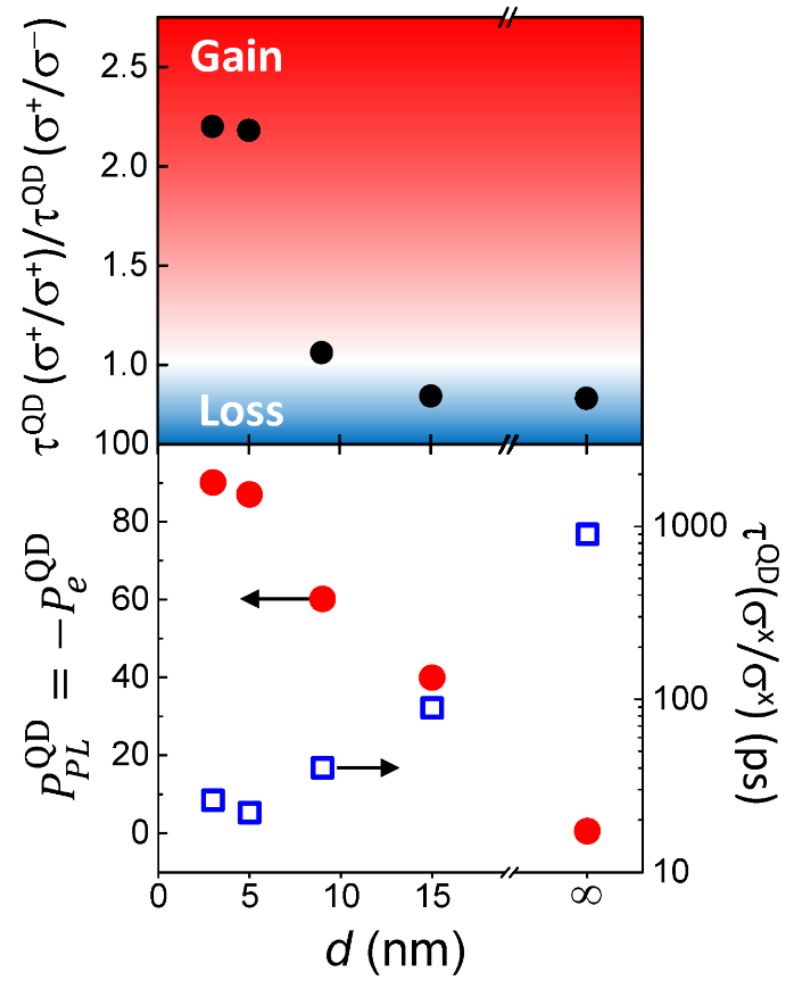

Fig. 4 | RT QD spin dynamics and effect of tunneling barrier thickness. a, Decay curves of the QD PL and $P_{P L}$ from the ref-QD and QD/GaNAs structure with $d=3 \mathrm{~nm}$, obtained by monitoring the $\sigma^{+}$and $\sigma^{-}$PL components (denoted by $\sigma^{+} / \sigma^{+}$and $\sigma^{+} / \sigma^{-}$, respectively) under $\sigma^{+}$excitation at $900 \mathrm{~nm}$. The dashed lines mark the effective PL decay times of the $\sigma^{+}$and $\sigma^{-}$PL components in the QD/GaNAs structure, i.e. $\tau^{Q D}\left(\sigma^{+} / \sigma^{+}\right)$and $\tau^{Q D}\left(\sigma^{+} / \sigma^{-}\right)$, which is affected by the remote spin filtering. The grey area represents the time window where the values of $\tau^{Q D}\left(\sigma^{+} / \sigma^{+}\right) / \tau^{Q D}\left(\sigma^{+} / \sigma^{-}\right)$shown in $\mathbf{b}$ were obtained. $\mathbf{b}, \tau^{Q D}\left(\sigma^{+} / \sigma^{+}\right) / \tau^{Q D}\left(\sigma^{+} / \sigma^{-}\right)$, $P_{P L}^{Q D}\left(=-P_{e}^{Q D}\right)$ and effective PL decay time $\tau^{Q D}\left(\sigma^{x} / \sigma^{x}\right)$ as a function of the tunneling barrier thickness $d$. The $P_{P L}^{Q D}\left(=-P_{e}^{Q D}\right)$ data were obtained from the cw PL experiments. $\tau^{Q D}\left(\sigma^{x} / \sigma^{x}\right)$ denotes the PL decay time measured under linearly polarized excitation and detection. The data points at $d \rightarrow \infty$ are taken from the ref-QD sample. The red (blue) shade indicates the region where spin filtering (spin relaxation) dominates. The data from the QD/GaNAs structure were taken from the sample with $\mathrm{N}$ composition of $1.5 \%$. All data were obtained at RT. 
a

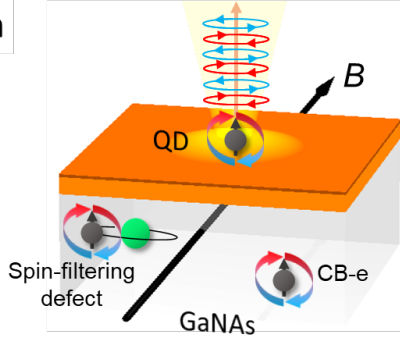

C

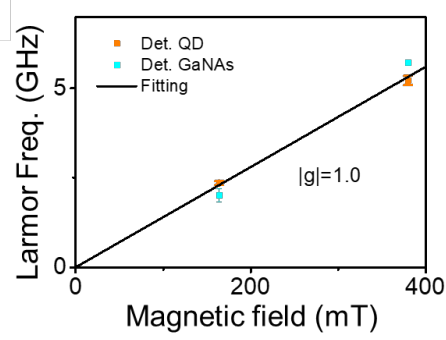

b
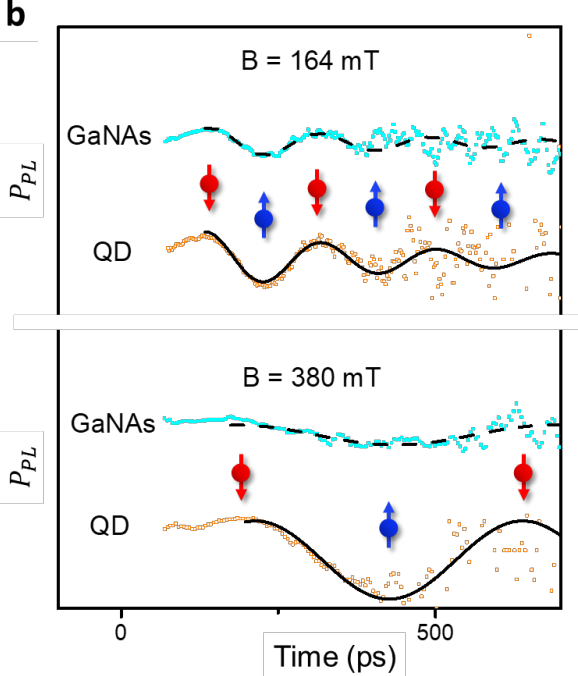

d
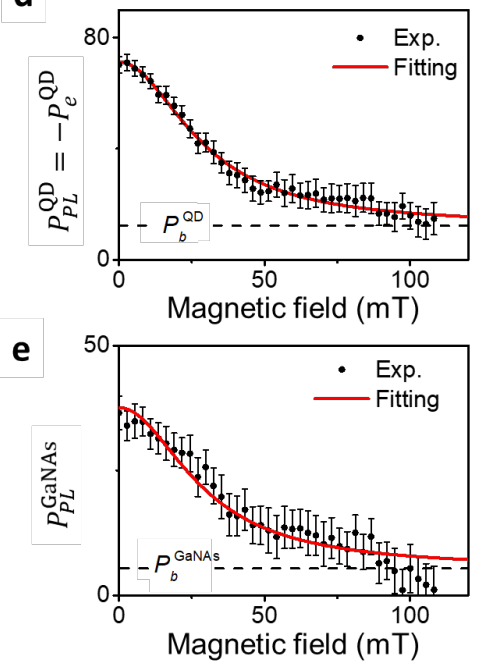

465

467

468

469

470

471

472

473

474

475

476

477

478

479

480

481

482

483

484

485

Fig. 5 | RT remote spin manipulation of QD electrons by the spin precession of CB and

defect electrons in GaNAs. a, Schematic illustration of the measurement configuration of $P_{e}^{Q D}\left(=-P_{P L}^{Q D}\right)$ in a transverse magnetic field B. b. Transient $P_{P L}$ by detecting QD and GaNAs emission from the GaNAs/QD structure under the influence of Larmor precession of the $\mathrm{CB}$ electrons in GaNAs at $\mathrm{B}=164 \mathrm{mT}$ and $380 \mathrm{mT}$, under pulsed $\sigma^{+}$excitation at 850 nm. The symbols are experimental data, whereas the solid and dashed lines are the fitting curves. The corresponding oscillations of the spin orientations of the QD and GaNAs electrons are also indicated. c, Larmor precession frequencies at the two magnetic fields based on the data in $\mathbf{b}$, together with the fitting curve with the specified g-value. The error bar is obtained from the fitting error using least-squares method. d, The Hanle curve obtained by detecting $P_{P L}^{Q D}$ within the spectral window of 1150-1180 nm. e, Hanle curve obtained by detecting $P_{P L}^{G a N A s}$ within the spectral window of 1000-1020 nm. The error bars in $\mathbf{d}$ and $\mathbf{e}$ are estimated from the statistical counting within each spectral window, which contains a sample number of 150 (d) and $100(\mathbf{e})$. In $\mathbf{d}$ and $\mathbf{e}, \mathrm{cw} \sigma^{+}$excitation at $800 \mathrm{~nm}$ (above the GaAs bandgap) was employed. The fitting of both Hanle curves yields a half width $B_{1 / 2}=29 \mathrm{mT}$.

The experimental values and the corresponding error bars are estimated from the mean value and standard deviation. 
Molecular beam epitaxy (MBE) fabrication. The studied sample structures, schematically

488

489

490

491

492

493

494

495

496

497

498

499

500

501

502

503

504

505

506

507

508

509

510

depicted in Fig.S1, were fabricated using a Veeco GEN20 MBE system on GaAs(100)

substrates. The system is equipped with SUMO-type effusion cells for the deposition of Al,

Ga and In, a valved cracker for As, and a radio-frequency Veeco UNI-Bulb plasma source for

N. For the epitaxy of the planar layers we used a growth rate of $0.5 \mu \mathrm{m} / \mathrm{h}$, while the InAs QDs were deposited at a rate of $0.05 \mu \mathrm{m} / \mathrm{h}$. The growth rates were calibrated prior to the fabrication of the samples. The $\mathrm{N}$ fluxes were calibrated using the incorporation model described in Ref.44.

The epitaxial process started with thermal removal of the substrate oxides and deposition of a GaAs buffer at a substrate temperature of $580{ }^{\circ} \mathrm{C}$. The GaNAs spin-filter layers were grown at a temperature of $400{ }^{\circ} \mathrm{C}$. Then the QDs were formed using selforganized Stranski-Krastanov growth mode by depositing 2.2 monolayer (ML) of InAs, and were capped with GaAs. The layered structure consisting of $20 \mathrm{~nm}$ GaNAs layer, GaAs tunneling barrier, 2.2 ML InAs QDs, and $50 \mathrm{~nm}$ GaAs layer capping the dots was repeated three times. We used four GaAs tunneling barrier thicknesses of 3, 5, 9 and $15 \mathrm{~nm}$. The structure was terminated with an additional 2.2 ML InAs QD layer used for assessing the density and the uniformity of the dotes. The structures were similar except for the $\mathrm{N}$ composition in the spin-filter layer, which was varied. We used four $\mathrm{N}$ compositions of $1.0 \%$, $1.5 \%, 2.0 \%$, or $2.6 \%$, leading to different alignments between the CB edge of the GaNAs layers and the QDs. In addition, the lh-hh VB splitting increases with increasing N composition; the splitting is estimated to be $19 \mathrm{meV}, 26 \mathrm{meV}$ and $35 \mathrm{meV}$ for the $\mathrm{N}$ compositions of $1.5 \%, 2.0 \%$, or $2.6 \%$, respectively ${ }^{45}$.

\section{Structural characterization by high-resolution x-ray diffraction and atomic force}


microscopy. After the epitaxy, the crystallography of the structures was probed using highresolution x-ray diffraction (XRD). Thus, we measured the (004) reflections using a Philips X'Pert Pro triple-axis XRD equipment and fitted the experimental data to a model using BEDE RADS software. As shown in Fig.S2, there is an excellent agreement between the XRD measurement $\omega-2 \theta$ data and the model fits for all the structures analyzed.

Atomic force microscopy (AFM) analysis revealed a QD density in the range of $4.5 \times 10^{10} / \mathrm{cm}^{2}$ to $6 \times 10^{10} / \mathrm{cm}^{2}$, with good size uniformity from sample to sample. Fig.S3 shows exemplary AFM images for the ref-QD sample and the QD/GaNAs sample with $1.5 \% \mathrm{~N}$ content, demonstrating similarity in QD density.

\section{Continuous-wave polarization-resolved optical and magneto-optical techniques. Optical} excitation was provided by a wavelength tunable Ti:Sapphire laser focused to a spot size of $\sim 2.2 \mu \mathrm{m}$ in diameter by using a microscope objective lens or $\sim 0.05 \mathrm{~mm}$ by using a conventional lens. The excitation power was over the range of $15-600 \mathrm{~mW}$ under $\mathrm{cw}-$ laser excitation and over the range of 3-70 $\mathrm{mW}$ under the pulsed-laser excitation (with a pulse width of $150 \mathrm{fs}$ and a repetition rate of $80 \mathrm{MHz}$ ). The laser line was tuned to $800-850 \mathrm{~nm}$ to optically generate electron spin polarization in GaAs, and $900-920 \mathrm{~nm}$ to generate it in GaNAs. In both cases, the excitation laser induced the band-to-band transitions involving both hh and lh VB states. The theoretical maximum electron spin polarization generated in GaAs or GaNAs was therefore $50 \%$, limited by the selection rules of the optical transitions. The PL emission was detected by an InGaAs CCD camera or a cooled Ge detector through a monochromator. For optical spin orientation experiments, a set of a linear polarizer and a quarter-wave plate were inserted into the excitation (detection) beam paths to create (detect) $\sigma^{+}$or $\sigma^{-}$polarized excitation (emission). In PL measurements at low temperatures, the samples were kept in a liquid-helium cooled cryostat. The measurements above room 
temperature were performed by placing the sample on a temperature controlled hot plate. The magneto-optical measurements were carried out with a calibrated electromagnet.

When the highest degree of electron spin polarization under the cw-laser excitation was obtained, the excitation power density was around $2.76 \mathrm{mW \mu m^{-2 }}$ that could theoretically lead to a steady-state density of around $n=7.1 \times 10^{17} \mathrm{~cm}^{-3}$ for photogenerated CB electrons in GaNAs. The actual density is expected to be lower if the loss due to reflection and scattering of the excitation light is considered. From the rate equation analysis of the excitation-power dependence of the GaNAs PL polarization and SDR ratio, as shown in Supplementary Figure S12, $n=3.3 \times 10^{16} \mathrm{~cm}^{-3}$ was deduced.

Time- and polarization-resolved optical and magneto-optical techniques. The timeresolved PL and PL polarization measurements were performed at RT under either circularly $\left(\sigma^{+}\right)$or linearly $\left(\sigma^{x}\right)$ polarized excitation from a mode-locked Ti:Sapphire laser. The excitation power was over the range of 3-70 $\mathrm{mW}$. The laser wavelength was set at $850 \mathrm{~nm}$ $(900 \mathrm{~nm})$ to generate initial electron spin polarization in GaAs (GaNAs). The excitation laser pulses had a spectral and temporal width of $10 \mathrm{~nm}$ and $100 \mathrm{fs}$, respectively. The full-width-athalf-maximum of the time response curve of the laser pulses was $6.7 \mathrm{ps}$. A home-made electromagnet was used to study the electron spin dynamics under spin precession in a transverse magnetic field. The strength of the magnetic field was calibrated by a standard Hall probe.

\section{Data availability}

All data generated or analyzed during this study are included in this published article (and its supplementary information files). 